\title{
Traction cart with adaptive drive
}

\author{
Konstantin Ivanov 1,2 , * \\ ${ }^{1}$ Chair “Control Systems for Aerospace Engineering “, Almaty University of Power Engineering and Telecommunications, Almaty, \\ Kazakhstan \\ ${ }^{2}$ Laboratory of Adaptive Mechanisms, Institute of Mechanics and Machine Science MON RK, Almaty, Kazakhstan
}

Email address:

ivanovgreek@mail.ru

\section{To cite this article:}

Konstantin Ivanov. Traction Cart with Adaptive Drive. American Journal of Mechanics and Applications. Special Issue: Adaptive Transmissions. Vol. 2, No. 6-1, 2014, pp. 29-34. doi: 10.11648/j.ajma.s.2014020601.16

\begin{abstract}
Wheel steams of an electric rolling stock are drive in rotation by traction engines which are placed in traction carts. Individual traction drive at which each wheel pair is drive in rotation by the traction engine has received wide application. In this drive the district speeds of wheels of wheel pairs appear different. It is necessary to create the control system of traction engines excluding their mismatch that is extremely difficult problem. Other variant of the decision of a problem consists in that the tooth gearing from the engine to wheel pair had the operated adjustable transfer ratio. Now under the patent of Kazakhstan it is developed the motor-wheel with an adaptive tooth gearing. Adaptive motor-wheel does not demand control system use. The use of adaptive motors-wheels will allow completely eliminating of mismatch of traction efforts of engines and sliding of wheels.
\end{abstract}

Keywords: Traction Cart, Motor-Wheel, Mismatch, Sliding, Adaptive Drive

\section{Introduction}

Wheel steams of an electric rolling stock are drive in rotation by traction engines which are placed in traction carts. The traction cart contains a frame, wheel steams, traction engines, traction transfers, spring suspension and lever-brake transfers.

Individual traction drive at which each wheel pair is drive in rotation by the traction engine has received wide application. The engine shaft is connected to an axis of wheel pair by tooth gearing which has the constant transfer ratio.

Peripheral speed of wheels of each wheel pair depends on radius of a wheel and the characteristic of the traction engine. Radiuses of wheels in different wheel steams are different because of discrepancies of manufacturing and different degree of deterioration. Characteristics of engines in different wheel steams are defined by electric parameters which coincide with some deviations. As a result district speeds of wheels of wheel pairs are different. It leads to mismatch of traction efforts when one wheel pair partially brakes another. The mismatch of traction efforts causes sliding of wheels on rails and decrease in factor of coupling. Spring fastening of the engine to a cart frame is not capable to eliminate a mismatch of traction efforts which continuously collects. Traction carts are inclined to wagging movement which causes the raised deterioration of rails and way frustration. Roughness of a way and turns are strengthen the sliding of wheels on rails. In the conditions of increase of speeds of a rolling stock the specified defects of movement essentially increase.

For elimination of sliding of wheels on rails it is necessary to provide identical peripheral speeds of wheels of all wheel pairs. For this purpose it is necessary to create the control system of traction engines excluding their mismatch that is extremely difficult problem.

Other variant of the decision of a problem consists in that the tooth gearing from the engine to wheel pair had the operated adjustable transfer ratio. Then it is necessary to create a control system of tooth gearings that also is a stubborn problem.

Recently there were patents for the elementary adaptive transfer [1, 2, 3 and 4] and the theory adaptive stepless adjustable transfer with two degrees of freedom $[5,6,7,8$ and 9] has been developed. Such transfer allows creating the small-sized adaptive motor-wheel possessing ideal adjustable to conditions of joint movement of all wheels of the transport machine without control system use.

\section{Traction Cart with Adaptive Drive}

Now under the patent of Kazakhstan it is developed the motor-wheel with an adaptive tooth gearing [4]. Adaptive motor-wheel [9] provides the independent adaptation to movement conditions only at the expense of properties of transfer mechanism. Adaptive motor-wheel does not demand 
control system use. Use of adaptive motors-wheels will allow completely eliminating of mismatch of traction efforts of engines and sliding of wheels.

Adaptive motor-wheel presented on Fig.1.

The basic parts of motor-wheel with the adaptive mechanical reductor (Fig. 1) are: 1. Frame. 2. Hub. 3. Alternating current induction motor 4. Adaptive small-size mechanical reductor. 5. Wheel rim. 6. Tyre The motor-wheel with the adaptive mechanical reductor has small sizes and provides adaptation of a wheel to variable technological loading without any control system.

The self-regulating wheelwork in the form of differential mechanism with the closed contour consisting from toothed wheels is presented at Fig. 2. The mechanism contains a frame 0 , input carrier ${ }^{H_{1}}$, input satellite 2 , block of the central wheels $1-4$, block of epicycle (ring) wheels 3-6, output satellite 5 and output carrier $\mathrm{H}_{2}$.

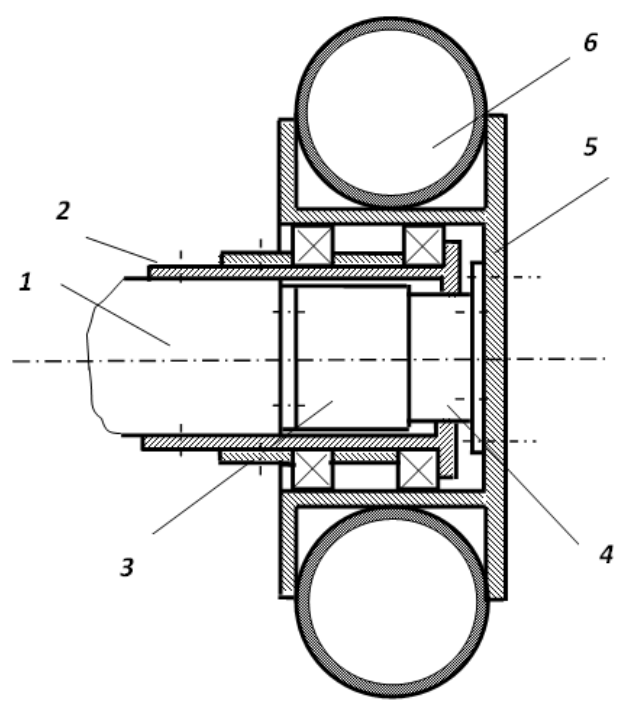

Figure 1. Adaptive motor-wheel

The adaptive transmission of the motor-wheel is simple on a design. It represents the gear differential mechanism with two degrees of freedom..

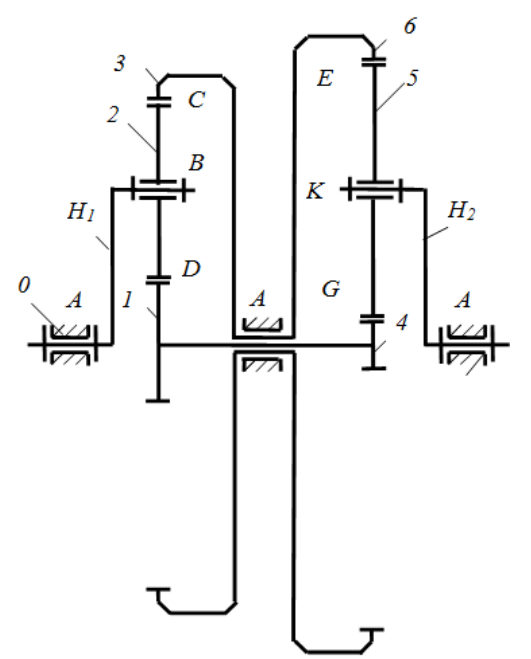

Figure 2. Adaptive transmission of motor-wheel
Mechanism has following structural groups with zero mobility:

1)Group $H_{2}-5$ containing two links $H_{2}$ and 5, two lowest kinematic pairs $A$ and $K$ and two higher kinematic pairs $E$ and $G$;

2)Group 3-6 containing one link 3-6, one lowest kinematic pair $A$ and one higher kinematic pair $C$;

3)Group 1-4 containing one link 1-4, one lowest kinematic pair $A$ and one higher kinematic pair $D$.

Mechanism has input structural group with two degrees of freedom containing links $H_{1}$ and 2 and two lowest kinematic pairs $A$ and $B$.

Basic features of the mechanism:

1)Input structural group with two degrees of freedom contains consistently connected two links,

2)Superposed forces $F_{H 1}$ and $R_{H 6}$ on links $H_{1}$ and $H_{2}$ act at the one line because radiuses of carriers $H_{1}$ and $H_{2}$ are equal.

3)Mechanism has the closed contour containing links 2, 3-6, $5,4-1$.

Let's do the power analysis of the mechanism when the output moment of resistance $M_{H 2}$ is set.

1)We determine reactions in kinematic pairs $E$ and $G$ of structural group $\mathrm{H}_{2}-5$ :

$$
\begin{gathered}
R_{65}=R_{H 2} / 2, R_{45}=R_{H 2} / 2 \\
\text { Here } R_{H 2}=M_{H 2} / r_{H 2} .
\end{gathered}
$$

2)We determine reactions in kinematic pairs $C$ and $D$ of structural groups 3-6 and 1-4:

$$
R_{23}=R_{65} \frac{r_{6}}{r_{3}} \quad R_{21}=R_{45} \frac{r_{4}}{r_{1}}
$$

Or

$$
\begin{gathered}
R_{32}=-R_{65} \frac{r_{6}}{r_{3}}=-R_{H 2} \frac{r_{6}}{2 r_{3}}, \\
R_{12}=-R_{45} \frac{r_{4}}{r_{1}}=-R_{H 2} \frac{r_{4}}{2 r_{1}} .
\end{gathered}
$$

3)We determine the input moment on a link 2

$$
M_{2}=-\left(R_{12}-R_{32}\right) r_{2} \cdot \text { Or } M_{2}=R_{H 2} \frac{r_{3} r_{4}-r_{1} r_{6}}{2 r_{1} r_{3}} r_{2} .
$$

4)We determine the input impellent on a link $H_{1}$

$$
F_{H 1}=-\left(R_{12}+R_{32}\right) \text {. Or }
$$




$$
F_{H 1}=R_{H 2} \frac{r_{1} r_{6}+r_{3} r_{4}}{2 r_{1} r_{3}}
$$

Each link of mechanism is statically counterbalanced in the absence of an inertial force. Hence the mechanism will move in regular intervals.

So, for overcoming of output resistance force it is necessary to use in input structural group 1-2 the input force $F_{H 1}$ on input link $H_{1}$ and the input moment $M_{2}$ on input link 2 . Then the number of input links (generalized co-ordinates) matches to number of degrees of freedom.

Let's present moment $M_{2}$ counterbalancing link 2 of input structural groups in an aspect the pairs of forces $P_{2}$ acting in points $C$ and $D$

$$
P_{2}=M_{2} / 2 r_{2}=R_{H 2} \frac{r_{3} r_{4}-r_{1} r_{6}}{4 r_{1} r_{3}}
$$

Then total forces $R_{C}$ and $R_{D}$ on a link 2 in points $C$ and $D$ will accept values:

$$
\begin{aligned}
R_{C}=R_{32}-P_{2} & =-R_{H 2} \frac{r_{6}}{2 r_{3}}-R_{H 2} \frac{r_{3} r_{4}-r_{1} r_{6}}{4 r_{1} r_{3}}= \\
& =-R_{H 2} \frac{r_{1} r_{6}+r_{3} r_{4}}{4 r_{1} r_{3}}, \\
R_{D}=R_{12}+P_{2} & =-R_{H 2} \frac{r_{4}}{2 r_{1}}+R_{H 2} \frac{r_{3} r_{4}-r_{1} r_{6}}{4 r_{1} r_{3}}= \\
& =-R_{H 2} \frac{r_{1} r_{6}+r_{3} r_{4}}{4 r_{1} r_{3}} .
\end{aligned}
$$

Or considering the formula (1) we will gain $R_{C}=R_{D}=-F_{H 1} / 2$. It means if on a link 2 of input structural group $H_{1}-2$ with two degrees of freedom to add reactions $R_{12}$ and $R_{32}$ transferred on it from the kinematic chain with the counterbalancing moment $M_{2}$ the link 2 will appear counterbalanced only one force $R_{H 1-2}=R_{12}+R_{32}=F_{H 1}$ applied in a point $B$.

But the same result will be gained on statics conditions if to consider that on input structural group $H_{1}-2$ one input driving moment $M_{H 1}=F_{H 1} r_{H 1}$ and one set input force $F_{H 1}$ matching to it counterbalancing reactions $R_{C}$ and $R_{D}$ transferred to this group act only. It means that the input structural group with two degrees of freedom is statically counterbalanced at presence only one generalized force $F_{H 1}$ (or the counterbalancing moment $M_{H 1}=F_{H 1} r_{H 1}$ ) on an input link $H_{1}$.

The gained conclusion defines paradox of mechanics - the input structural group with two degrees of freedom in the kinematic chain with the closed contour is statically definable (counterbalanced) at presence only one generalized force.

The paradox of mechanics provides static definability of the kinematic chain with two degrees of freedom at presence only one input. Thus the examined kinematic chain with two degrees of freedom but with one input is the mechanism.

Let's will prove it analytically.

With the input structural group having only one input impellent it is possible to examine the kinematic chain as the converted kinematic chain. The converted kinematic chain is equivalent on acting forces to initial kinematic chain with the input counterbalancing moment on a link 2 .

Reactions are transferred to intermediate links 1-4 and 3-6: $R_{23}=R_{21}=F_{H 1} / 2$ from the input satellite 2 and $R_{54}=R_{56}=R_{H 2} / 2$ from the output satellite 5 .

The balance of superposed forces in the kinematic chain takes place

$$
M_{H 1}+M_{H 2}=0 \text {. }
$$

At equality of magnitudes of the external moments $M_{H 1}=M_{H 2}$ we have $R_{H 2}=F_{H 1} r_{H 1} / r_{H 2}$.

As a result the link 1-4 will appear under the action of the unbalanced moment

$$
M_{1-4}=R_{21} r_{1}-R_{54} r_{4}=\frac{F_{H 1}}{2}\left(\frac{r_{H 2} r_{1}-r_{H 1} r_{4}}{r_{H 2}}\right) .
$$

The link 3-6 will appear under the action of the unbalanced moment

$$
M_{3-6}=R_{23} r_{3}-R_{56} r_{6}=\frac{F_{H 1}}{2}\left(\frac{r_{H 2} r_{3}-r_{H 1} r_{6}}{r_{H 2}}\right) \text {. }
$$

Let's substitute in formulas (3), (4) values $r_{H 1}=\left(r_{1}+r_{3}\right) / 2, r_{H 2}=\left(r_{4}+r_{6}\right) / 2$ we will gain

$$
\begin{aligned}
& M_{1-4}=\frac{F_{H 1}}{2}\left(\frac{r_{1} r_{6}-r_{3} r_{4}}{r_{4}+r_{6}}\right) . \\
& M_{3-6}=\frac{F_{H 1}}{2}\left(\frac{r_{3} r_{4}-r_{1} r_{6}}{r_{4}+r_{6}}\right) .
\end{aligned}
$$

From here the equation follows $M_{1-4}=-M_{3-6}$. That is

$$
M_{1-4}+M_{3-6}=0 \text {. }
$$

The formula (7) defines balance of internal forces.

Thus for wheelwork internal forces on each intermediate link 1-4 and 3-6 are led to the moments $M_{1-4}$ and $M_{3-6}$ on 
each intermediate block of wheels which are unbalanced on conditions of statics. However for the mechanism as a whole according to the formula (7) balance of internal forces (moments) takes place. Balance of superposed forces (moments) by formula (2) simultaneously takes place. Hence all mechanism is in balance.

\section{The Analysis of Motion of the Kinematic Chain}

Let's examine regularities of interacting of parameters on the motion of the kinematic chain by means of a principle of virtual works.

This condition of balance contains not only acting forces but also possible displacements (speeds). It is used for the kinematic chain moving under the action of forces.

According to a principle of possible works - the sum of works (powers) of all external and internal forces is equal to zero.

Let's make for each satellite an equilibrium equation by a principle of possible works (powers). We will gain for the satellite 2

$$
F_{H 1} v_{H 1}+R_{12} v_{1}+R_{32} v_{3}=0
$$

Let's express linear speeds of links through angular speeds. We will gain

$$
\begin{gathered}
F_{H 1} \omega_{H 1} r_{H 1}+R_{12} \omega_{1} r_{1}+R_{32} \omega_{3} r_{3}=0 . \text { Or } \\
M_{H 1} \omega_{H 1}+M_{1} \omega_{1}+M_{3} \omega_{3}=0 .
\end{gathered}
$$

Analogously for the satellite 5 (with the account $\left.\omega_{1}=\omega_{4}, \omega_{3}=\omega_{6}\right)$

$$
M_{H 2} \omega_{H 2}+M_{4} \omega_{1}+M_{6} \omega_{3}=0 \text {. }
$$

As satellites are a part of the mechanism we will add the made expressions for satellites. We will gain a condition of interacting of parameters of the mechanism as a whole

$$
\begin{gathered}
M_{1} \omega_{1}+M_{3} \omega_{3}+M_{4} \omega_{1}+M_{6} \omega_{3}+ \\
+M_{H 1} \omega_{H 1}+M_{H 2} \omega_{H 2}=0 \\
\operatorname{Or}\left(M_{1}-M_{4}\right) \omega_{1}+\left(M_{3}-M_{6}\right) \omega_{3}+ \\
+M_{H 1} \omega_{H 1}+M_{H 2} \omega_{H 2}=0
\end{gathered}
$$

Let's mark out

$$
M_{1-4}=M_{1}-M_{4}, M_{3-6}=M_{3}-M_{6} \text {. }
$$

We will gain

$$
M_{1-4} \omega_{1}+M_{3-6} \omega_{3}+
$$

$$
+M_{H 1} \omega_{H 1}+M_{H 2} \omega_{H 2}=0
$$

In the presence of balance the sum of powers of superposed forces is equal to zero

$$
M_{H 1} \omega_{H 1}+M_{H 2} \omega_{H 2}=0 .
$$

Then from (11) for internal forces

$$
M_{1-4} \omega_{1}+M_{3-6} \omega_{3}=0 \text {. }
$$

For a particular case examined above at $M_{H 1}=-M_{H 2}$ and $M_{1-4}=-M_{3-6}$ the conditions (11) and (12) are carried out if $\omega_{H 1}=\omega_{H 2}=\omega_{3}=\omega_{4}$ - the kinematic chain moves as a single whole without internal mobility of links.

Further let's examine the common case of balance in the movement at

$$
M_{H 1} \neq M_{H 2}, \omega_{H 1} \neq \omega_{H 2} .
$$

Let's express the internal moments on blocks of wheels 1-4 and 3-6 through superposed forces (moments)

$$
\begin{gathered}
M_{1-4}=M_{1}-M_{4}= \\
=R_{12} r_{1}-R_{45} r_{4}=\frac{F_{H 1}}{2} r_{1}-\frac{R_{H 2}}{2} r_{4} . \mathrm{Or} \\
M_{1-4}=\frac{M_{H 1}}{2 r_{H 1}} r_{1}-\frac{M_{H 2}}{2 r_{H 2}} r_{4} . \\
M_{3-6}=M_{3}-M_{6}= \\
=R_{32} r_{3}-R_{65} r_{6}=\frac{F_{H 1}}{2} r_{3}-\frac{R_{H 2}}{2} r_{6} . \text { Or } \\
M_{3-6}=\frac{M_{H 1}}{2 r_{H 1}} r_{3}-\frac{M_{H 2}}{2 r_{H 2}} r_{6} .
\end{gathered}
$$

From (12) taking into account signs of the moments

$$
M_{H 1}=M_{H 2} \omega_{H 2} / \omega_{H 1}
$$

Let's mark out $u_{21}=\omega_{H 2} / \omega_{H 1}$ - the transfer ratio of the mechanism. Then we have $M_{H 1}=M_{H 2} u_{12}$. After substitution of value of the moment $M_{H 1}$ in formulas (14) and (15) we will gain

$$
M_{1-4}=\frac{M_{H 2}}{2} \cdot \frac{u_{21} r_{H 2} r_{1}-r_{H 1} r_{4}}{r_{H 1} r_{H 2}}
$$

$$
M_{3-6}=\frac{M_{H 2}}{2} \cdot \frac{u_{21} r_{H 2} r_{3}-r_{H 1} r_{6}}{r_{H 1} r_{H 2}}
$$


Let's substitute in the formula (13) values of the moments $M_{1-4}$ and $M_{3-6}$. After abbreviation on $M_{H 2} / 2 r_{H 1} r_{H 2}$ we will gain

$$
\begin{gathered}
\left(u_{21} r_{H 2} r_{1}-r_{H 1} r_{4}\right) \omega_{1}+ \\
+\left(u_{21} r_{3} r_{H 2}-r_{H 1} r_{6}\right) \omega_{3}=0 .
\end{gathered}
$$

Let's substitute in the formula (18) value $u_{21}=\omega_{H 2} / \omega_{H 1}$. With the account $v_{H 2}=\omega_{H 2} r_{H 2}, v_{H 1}=\omega_{H 1} r_{H 1}$, $v_{1}=\omega_{1} r_{1}, v_{4}=\omega_{4} r_{4}, v_{3}=\omega_{3} r_{3}, v_{6}=\omega_{3} r_{6}$ we will gain

$$
\begin{gathered}
\frac{v_{1} v_{H 2}-v_{4} v_{H 1}}{\omega_{H 1}}+\frac{v_{3} v_{H 2}-v_{6} v_{H 1}}{\omega_{H 1}}=0 . \text { Or } \\
v_{H 2}\left(v_{1}+v_{3}\right)-v_{H 1}\left(v_{4}+v_{6}\right)=0 .
\end{gathered}
$$

From a picture of speeds of a wheelwork (Fig. 3) it is visible

$$
v_{1}+v_{3}=2 v_{H 1}, v_{4}+v_{6}=2 v_{H 2} \text {. }
$$

After substitution these values in the formula (19) we will gain

$$
2 v_{H 2} v_{H 1}-2 v_{H 2} v_{H 1}=0 \text {, That is } 0=0 \text {. }
$$

It means that the condition of balance of internal forces (13) by a principle of possible work is carried out if the condition of balance (12) for superposed forces is satisfied.

Thus for the kinematic chain balance of internal forces in the presence of unbalanced separately links 3 and 4 takes place. But this balance takes place only in the motion.

The executed researches of kinematics and the power analysis of the kinematic chain with two degrees of freedom allow making following conclusions:

1) Power analysis of a chain is carried out as the solution of a direct problem of dynamics - on the set motion to define forces. The set parameters are input angular speed $\omega_{H 1}$ and output moment of resistance $M_{H 2}$. However the formula (12) allows setting the input driving moment also $M_{H 1}$. The setting of the external moments $M_{H 1}$ and $M_{H 2}$ (on which internal forces are defined) characterizes static definability of the kinematic chain. According to the formula (12) static (power) definability of the kinematic chain takes place on the motion.

2) Formula (12) characterizes the kinematic definability of the kinematic chain with two degrees of freedom. At the set input speed $\omega_{H 1}$ and the set external moments $M_{H 1}$ and $M_{H 2}$ the formula (12) allows determining an output speed $\omega_{H 2}$. Through the speeds $\omega_{H 1}$ and $\omega_{H 2}$ the speeds of intermediate links are determined.

3) Formula (12) connecting the kinematic and power parameters of the kinematic chain with two degrees of freedom represents additional constraint which imposes the gearing chain on motion of links. This additional constraint will neutralize one degree of freedom and at presence only one input will convert a chain to the mechanism.

4) From the formula (12) follows

$$
\omega_{H 2}=\frac{M_{H 1} \omega_{H 1}}{M_{H 2}} .
$$

The formula (20) defines effect of power adaptation: at a constant input power $N_{H 1}=M_{H 1} \omega_{H 1}$ the output speed $\omega_{H 2}$ inversely proportional to external resistance moment. The effect of power adaptation characterizes the major property for machines with variable technological resistance ability independently and continuously to adapt for variable technological loading.

The kinematic chain with two degrees of freedom having only one input is gear continuously variable transmission.

\section{Conclusion}

Use of the found regularities allows to create the motors-wheels possessing property of mechanical adaptation to variable technological loading. The adaptive mechanism provides possibility of motion of output link with a speed inversely proportional moment of resistance at a constant input power.

The adaptive mechanical wheelwork uses the effect of power adaptation allowing automatically changing the transfer ratio. It allows simplifying a design, to reduce transmission sizes, to raise its reliability and efficiency.

The motor-wheel with the adaptive electric drive which is containing the electric motor and the small-size mechanical transmission ideally adapts for variable technological loading without use of any control systems. For example, the leading motors-wheels installed on an electric motor car will provide full adaptation to motion on turn with matching different rotation speeds of wheels.

Future enhancement consists in synthesis of a wheelwork of a motor-wheel on a condition of overcoming of the set maximum moment of resistance.

The traction cart with adaptive motor-wheels will procure the motion of rolling stock with very high efficiency without a sledding, a wagging and power waste.

\section{References}

[1] Harries John. Power transmission system comprising two sets of epicyclic gears. Patent of Great Britain GB2238090 (A.) 1991, $11 \mathrm{p}$.

[2] Ivanov K.S., Yaroslavtseva E.K. Way of automatic and continuous change of a twisting moment and speed of twirl of the output shaft depending on a tractive resistance and the device for its realization. The description of the invention to the patent application of Russia RU 2007139258/11 (042965), the solution on output of the patent from March, 25th, 2010. 28 p. 
[3] Konstantin S. Ivanov, Almaty, KAZ - Owner of the registered sample. The name - Device of automatic and continuous change of a twisting moment - and changes of a corrected speed of output shaft depending on a tractive resistance. The deed on registration of the registered sample № 202012101 273.1. Day of Registration 02.05.2012. The German patent and firm establishment. Federal Republic Germany. 2012. 12 p.

[4] Ivanov K.S., Sagitov P. I, Актаев E.T.Motor-wheel. Innovative patent RK №26372 from 27.02.2012. Kazpatent. Astana. 2012. $10 \mathrm{p}$.

[5] Ivanov K.S. The simplest automatic transfer box. WCE 2010. World Congress on Engineering 2010 (ICME) London, UK. 2010. - P. $1179-1184$.

[6] Ivanov K.S. Synthesis of Toothed Continuously Variable Transmission (CVT). Mechanism, Transmissions and
Applications. Mechanism and Machine Science 3. Springer. 2012. P. $265-272$.

[7] Ivanov K.S. Synthesis of Toothed Continuously Variable Transmission (CVT). Mechanism, Transmissions and Applications. Mechanism and Machine Science 3. Springer. 2012. P. $265-272$.

[8] Ivanov K.S. Paradox of mechanics - a basis of creation CVT. Transactions of 2-d IFToMM Asian Conference on Mechanisms and Machines Science. November 7-10, 2012, Tokyo, Japan. P. $245-264$.

[9] Ivanov K.S. Self-Adjusting Motor-Wheel with CVT. International Journal of Engineering and Innovanive Technology (IJEIT). Volume 2, Issue 4. Florida. USA. 2012. PP189 - 195. 